\title{
Violência contra a pessoa idosa: análise das notificações realizadas no setor saúde - Brasil, 2010
}

\author{
Violence against the elderly: analysis of the reports \\ made in the health sector - Brazil, 2010
}

\author{
Márcio Dênis Medeiros Mascarenhas ${ }^{1}$ \\ Silvânia Suely Caribé de Araújo Andrade ${ }^{2}$ \\ Alice Cristina Medeiros das Neves ${ }^{1}$ \\ Ana Amélia Galas Pedrosa ${ }^{3}$ \\ Marta Maria Alves da Silva ${ }^{1}$ \\ Deborah de Carvalho Malta ${ }^{1}$
}

${ }^{1}$ Coordenação Geral de

Vigilância de Doenças e

Agravos Não

Transmissíveis

(CGDANT), Secretaria de

Vigilância em Saúde (SVS),

Ministério da Saúde (MS).

Esplanada dos Ministérios

Bloco G Edifício Sede Sala

142. 70058-900 Brasília

DF.marcio.mascarenhas@

saude.gov.br

${ }^{2}$ Programa de Pós-

Graduação em Ciências

Médicas, Universidade

Estadual de Campinas,

Campinas-SP

${ }^{3}$ Fundação Municipal de

Saúde, Teresina-PI

Ministério da Saúde,

Secretaria de Vigilância em

Saúde

\begin{abstract}
The scope of this study was to describe reports of violence against the elderly ( $e$ " 60 years) reported in the Information System for Notifiable Diseases - net version (Sinan Net) in 2010. We conducted a descriptive, retrospective study with data analyzed by Stata version 11. We estimated proportion ratios $(P R)$ of violence according to selected variables. Of the 3,593 reports of violence against the elderly, 52.3\% were against females. Physical violence was significantly more frequent against males $(P R=0.82)$ in the group aged 60 to 69 years, out of the home, committed by offenders who were not sons and were suspected of drinking alcohol. Psychological violence was more common among elderly people $(P R=2.17)$, in the home, inflicted by sons, with suspected chronic alcohol abuse. Negligence was predominant among females ( $P R=1.24$ ), in the group above 70 years of age, in the home, recurrently perpetrated by sons. Sexual violence was more common against females $(P R=5.21)$, by offenders who were not children, but who consumed alcohol. The knowledge of the different manifestations of violence against the elderly supports actions to combat them, identifying characteristics of vulnerability in which support networks may intervene.
\end{abstract}

Key words Violence, Abuse, Elderly, Epidemiological surveillance
Resumo O objetivo do estudo foi descrever notificações de violência contra os idosos ( $\geq 60$ anos) captadas pelo Sistema de Informação de Agravos de Notificação - versão net (Sinan Net) em 2010. Realizou-se estudo descritivo, retrospectivo, com dados analisados no Stata versão 11. Estimaramse razões de proporção $(R P)$ de violência segundo variáveis selecionadas. Das 3.593 notificações de violência contra idosos, 52,3\% eram referentes ao sexo feminino. A violência física foi significativamente mais frequente no sexo masculino $(R P=$ 0,82), no grupo com 60 a 69 anos, fora do domicílio, praticada por agressores que não eram filhos, com suspeita de ingestão de bebida alcoólica. A violência psicológica foi mais frequente entre idosas $(R P=2,17)$, no domicílio, infligida pelos filhos, com suspeita de uso de bebida alcoólica e de maneira crônica. A negligência predominou no sexo feminino $(R P=1,24)$, no grupo a partir de 70 anos, no domicílio, perpetrada pelos filhos e recorrente. A violência sexual foi mais comum no sexo feminino $(R P=5,21)$, por agressores que não eram filhos, mas que consumiram bebida alcoólica. O conhecimento das diferentes manifestações da violência contra idosos subsidia ações para o seu enfrentamento, identificando características de vulnerabilidade onde as redes de apoio podem intervir.

Palavras-chave Violência, Maus-tratos, Idoso, Vigilância epidemiológica 


\section{Introdução}

O envelhecimento populacional é um fenômeno verificado por meio do aumento na proporção de pessoas idosas (60 anos e mais) resultante do declínio da fecundidade, da queda nas taxas de mortalidade e do aumento da expectativa de vida $^{1,2}$. Segundo dados dos últimos censos realizados pelo Instituto Brasileiro de Geografia e Estatística $(\mathrm{IBGE})^{3}$, a composição etária do Brasil vem apresentando constante aumento da proporção de idosos: em 1980 existiam 7,2 milhões de pessoas com idade a partir de 60 anos, enquanto em 2010 este contingente populacional chegou a somar 20,6 milhões. Ou seja, o número de pessoas idosas aumentou 2,9 vezes em 40 anos, chegando a representar $10,8 \%$ da população brasileira em 2010.

Além dos fenômenos inerentes ao processo de envelhecimento, como as modificações fisiológicas e patologias consideradas típicas da terceira idade, a pessoa idosa também está susceptível ao fenômeno da violência. Trata-se de um problema com consequências devastadoras para os idosos, pois acarreta baixa qualidade de vida, estresse psicológico, falta de segurança, lesões e traumas, bem como o aumento da morbidade e mortalidade ${ }^{4,5}$. A violência contra o idoso é um ato (único ou repetido) ou omissão que lhe cause dano ou aflição e resulta, na maioria das vezes, em sofrimento, lesão, dor, omissão ou perda dos direitos humanos e redução da qualidade de vida do idoso ${ }^{6,7}$.

Observada particularmente nas culturas ocidentais, a violência contra o idoso é uma manifestação independente do nível socioeconômico e se justifica pela concepção socialmente aceita de que o idoso é considerado como algo descartável ou um peso social. Trata-se de um segmento da população pelo qual se nutre um "desejo social de morte", que se expressa, sobretudo, nos conflitos intergeracionais, maus-tratos e negligências nas esferas político-institucionais e, marcadamente, no âmbito do domicílio ${ }^{8}$.

Em 2009, as causas externas (acidentes e violência) ocuparam a sexta posição entre os óbitos de idosos no Brasil, totalizando 21.453 mortes, das quais 1.929 foram devidas a agressões (homicídios). O risco de morte por homicídio entre idosos foi de 9,9 por 100 mil idosos, sendo que o risco de morte entre homens (19/100 mil) foi sete vezes o das mulheres $(2,7 / 100 \mathrm{mil})$ na mesma faixa etária ${ }^{9}$.

Dada sua importância como problema de saúde pública, a violência contra a pessoa idosa tornou-se objeto de vigilância epidemiológica no
Brasil a partir de 2006, por meio da implantação do Sistema de Vigilância de Violências e Acidentes (VIVA) ${ }^{10}$, atendendo ao que determina a Lei $\mathrm{n}^{\circ}$. 10.741/2003 (Estatuto do Idoso), artigo 19: "os casos de suspeita ou confirmação de maustratos contra idoso serão obrigatoriamente comunicados pelos profissionais de saúde"11. Recentemente, a redação anterior foi alterada pela Lei no. 12.461/2011: "os casos de suspeita ou confirmação de violência praticada contra idosos serão objeto de notificação compulsória pelos serviços de saúde públicos e privados à autoridade sanitária, bem como serão obrigatoriamente comunicados por eles"12.

O VIVA possui dois componentes: vigilância contínua e vigilância por inquérito. $\mathrm{O}$ primeiro componente consiste na captação contínua de dados sobre violência doméstica, sexual e/ou outras violências pelos serviços de saúde ${ }^{10}$. No período de 2006 a 2008, os dados do VIVA Contínuo eram digitados no aplicativo Epi Info 3.5.1 (Centers for Disease Control and Prevention, Atlanta, Estados Unidos). A partir de 2009, os dados passaram a integrar o Sistema de Informação de Agravos de Notificação - versão Net (Sinan Net), o que facilitou a expansão do VIVA e a obrigatoriedade da notificação de violência em serviços sentinela por meio da Portaria MS/GM n ${ }^{\circ}$. 2.472/ $2010^{13}$. Em 2011, por meio da Portaria MS/GM $n^{\circ}$. 104/2011, a violência foi incluída na relação de doenças e agravos de notificação compulsória em todo o território nacional, ou seja, passou a ser um agravo de notificação universal em todos os serviços de saúde do Brasil ${ }^{14}$.

Frente a esta nova realidade demográfica e epidemiológica, na qual se destaca a violência contra a pessoa idosa, é necessário analisar e divulgar o perfil das notificações realizadas nos serviços de saúde. Portanto, o presente artigo tem por objetivo descrever as notificações de violência contra a pessoa idosa captadas pelo Ministério da Saúde no ano de 2010.

\section{Métodos}

Estudo descritivo, retrospectivo, realizado com dados obtidos no Sistema de Informação de Agravos de Notificação - versão Net (Sinan Net) abrangendo as notificações de violência doméstica, sexual e/ou outras violências na população de idosos ( $\geq 60$ anos) realizadas no período de 01 de janeiro a 31 de dezembro de 2010.

Para os fins de notificação no Sinan Net, violência é considerada como "o uso intencional de 
força física ou do poder, real ou em ameaça, contra si próprio, contra outra pessoa, ou contra um grupo ou uma comunidade que resulte ou tenha possibilidade de resultar em lesão, morte, dano psicológico, deficiência de desenvolvimento ou privação"6.

Segundo diretrizes do Ministério da Saúde ${ }^{15}$, são passíveis de notificação de violência contra a pessoa idosa casos suspeitos ou confirmados de:

Violência física (sevícia física, maus-tratos físicos ou abuso físico): atos violentos, nos quais se faz uso da força física de forma intencional, não acidental, com o objetivo de ferir, lesar, provocar dor e sofrimento ou destruir a pessoa, deixando, ou não, marcas evidentes no seu corpo.

Violência psicológica: toda forma de rejeição, depreciação, discriminação, desrespeito, cobrança exagerada, punições humilhantes e utilização da pessoa para atender às necessidades psíquicas de outrem.

Tortura: ato de constranger alguém com emprego de força ou grave ameaça, causandolhe sofrimento físico ou mental com a finalidade de obter informação, declaração ou confissão da vítima ou de terceira pessoa, provocar ação ou omissão de natureza criminosa, em razão de discriminação racial ou religiosa.

Violência sexual: qualquer ação na qual uma pessoa, valendo-se de sua posição de poder e fazendo uso de força física, coerção, intimidação ou influência psicológica, com uso ou não de armas ou drogas, obriga outra pessoa - de qualquer sexo - a ter, presenciar, ou participar, de alguma maneira, de interações sexuais ou a utilizar, de qualquer modo, a sua sexualidade, com fins de lucro, vingança ou outra intenção.

Tráfico de seres humanos: inclui o recrutamento, o transporte, a transferência, o alojamento de pessoas, recorrendo à ameaça, ao rapto, à fraude, ao engano, ao abuso de autoridade, ao uso da força ou a outras formas de coação, ou à situação de vulnerabilidade, para exercer prostituição, ou trabalho sem remuneração, escravo ou de servidão, ou para remoção e comercialização de órgãos, com emprego ou não de força física.

Violência financeira (econômica): ato de violência que implica dano, perda, subtração, destruição ou retenção de objetos, documentos pessoais, bens e valores de outrem.

Negligência (abandono): omissão pela qual se deixou de prover as necessidades e os cuidados básicos para o desenvolvimento físico, emocional e social de outrem.

Intervenção legal: ato violento praticado durante intervenção por agente legal público, isto é, representante do Estado, polícia ou de outro agente da lei no uso da sua função.

Os dados foram captados por meio da Ficha de notificação/investigação individual de violência doméstica, sexual e/ou outras violências, que contém variáveis sobre: vítima/pessoa atendida, ocorrência, tipologia da violência, consequências da violência, lesão, provável agressor, evolução e encaminhamentos. A ficha é preenchida nos serviços de saúde e outras fontes notificadoras (asilos, centros de convivência, centros de referência para vítimas de violência) e os dados são digitados no Sinan Net no nível municipal e transferidos para as esferas estadual e federal para compor a base de dados nacional.

As notificações de violência contra idosos foram analisadas segundo: características demográficas das vítimas (sexo, idade, raça/cor da pele, escolaridade, situação conjugal, presença de deficiência ou transtorno); características da ocorrência (local, violência de repetição, natureza da lesão, parte do corpo atingida, evolução); tipo de violência e meio de agressão; características do agressor (sexo, tipo, suspeita de consumo de bebida alcoólica). Os registros foram importados do Sinan Net e as análises estatísticas foram processadas no programa Stata versão 11 (Stata Corp., College Station, Estados Unidos). Foram calculadas proporções e realizadas comparações entre as variáveis qualitativas por meio do teste do qui-quadrado $\left(\chi^{2}\right)$, com nível de significância de $5 \%$. Estimaram-se razões de proporção (RP) e respectivos intervalos de confiança de 95\% (IC95\%) para os tipos de violência mais frequentes segundo variáveis selecionadas.

O projeto de implantação do VIVA foi aprovado pela Comissão Nacional de Ética em Pesquisa (CONEP) do Conselho Nacional de Saúde. Por se tratar de uma ação permanente de vigilância epidemiológica instituída pelo Ministério da Saúde em todo o território nacional, não foi emitido termo de consentimento livre e esclarecido. Porém, foram garantidos o anonimato e a confidencialidade das informações nos registros para preservar a identidade dos indivíduos que compunham a base de dados analisada.

\section{Resultados}

Foram registradas 3.593 notificações de violência contra idosos nos estabelecimentos notificadores do Sinan Net no ano 2010. As notificações eram procedentes de 524 municípios brasileiros. A mediana de notificações foi igual a 44, varian- 
do de uma notificação em Sergipe a 1.073 notificações em São Paulo, estado responsável por aproximadamente $30 \%$ de todas as notificações. Não houve notificações no estado do Pará (dados não apresentados).

As características demográficas dos idosos vítimas de violência encontram-se detalhadas na Tabela 1. Do total de casos notificados, 52,3\% referiam-se a vítimas do sexo feminino. A média de idade dos idosos vítimas de violência foi de 71,1 anos (desvio-padrão $=8,8$ anos), variando de 60 a 109 anos. Aproximadamente metade dos idosos tinha de 60 a 69 anos de idade (50,2\%), $64,8 \%$ declararam-se brancos, $87,9 \%$ referiram ter concluído até 8 anos de estudo, 58,1\% não tinham companhia marital e $21,8 \%$ informaram possuir algum tipo de deficiência ou transtorno. Observou-se diferença significativa na distribuição das notificações segundo faixa etária, cor da pele e situação conjugal: a proporção de idosos com idade a partir de 70 anos, de pele branca e que viviam sem parceria conjugal foi maior no sexo feminino. Não foi observada diferença sig- nificativa para as variáveis escolaridade e presença de deficiência ou transtornos.

A maioria dos episódios de violência contra idosos ocorreu no domicílio $(78,8 \%)$ e mais da metade das vítimas referiu que já tinha sido violentada previamente $(53,6 \%)$. As mulheres foram vítimas de violência no domicílio e de violência de repetição em maior proporção comparativamente aos homens. Aproximadamente $11 \%$ das notificações apontavam a ocorrência de lesões autoprovocadas, porém não foi possível saber se havia intenção de suicídio. Essa informação foi significativamente mais prevalente no sexo masculino $(13,1 \%)$. Quanto à natureza da lesão, predominaram os eventos que resultaram em lesões do tipo corte/laceração/amputação $(25,5 \%)$, com ocorrência superior entre os homens, e contusão/ entorse/luxação (22,6\%), com maior frequência entre as mulheres. A região da cabeça/pescoço $(44,3 \%)$ foi o principal alvo das lesões resultantes de violência contra idosos, principalmente entre os homens (50,2\%), enquanto as lesões em membros superiores $(19,5 \%)$ foram mais frequentes

Tabela 1. Notificações de violência contra idosos segundo características demográficas por sexo. Brasil, 2010.

\begin{tabular}{|c|c|c|c|c|c|c|c|}
\hline \multirow[b]{2}{*}{ Características } & \multicolumn{2}{|c|}{$\begin{array}{c}\text { Feminino } \\
(\mathrm{N}=1.880 \\
52,3 \%)\end{array}$} & \multicolumn{2}{|c|}{$\begin{array}{c}\text { Masculino } \\
(\mathrm{N}=1.713 \\
47,7 \%)\end{array}$} & \multicolumn{2}{|c|}{$\begin{array}{c}\text { Total } \\
(\mathrm{N}=3.593 \\
100 \%)\end{array}$} & \multirow[b]{2}{*}{ p-valor } \\
\hline & $\mathbf{N}$ & $\%$ & $\mathbf{N}$ & $\%$ & $\mathbf{N}$ & $\%$ & \\
\hline Faixa etária (anos) & & & & & & & 0,007 \\
\hline $60-69$ & 903 & 48,0 & 900 & 52,5 & 1.803 & 50,2 & \\
\hline$\geq 70$ & 977 & 52,0 & 813 & 47,5 & 1.790 & 49,8 & \\
\hline Total & 1.880 & 100,0 & 1.713 & 100,0 & 3.593 & 100,0 & \\
\hline Raça/cor da pele ${ }^{a}$ & & & & & & & 0,007 \\
\hline Brancos & 1.005 & 67,1 & 798 & 62,2 & 1.803 & 64,8 & \\
\hline Negros (pretos+pardos) & 493 & 32,9 & 485 & 37,8 & 978 & 35,2 & \\
\hline Total $^{\mathrm{b}}$ & 1.519 & 100,0 & 1.301 & 100,0 & 2.820 & 100,0 & \\
\hline Escolaridade (anos de estudo) & & & & & & & 0,597 \\
\hline$\leq 8$ & 768 & 87,5 & 703 & 88,3 & 1.471 & 87,9 & \\
\hline$\geq 9$ & 110 & 12,5 & 93 & 11,7 & 203 & 12,1 & \\
\hline Total $^{\mathrm{b}}$ & 878 & 100,0 & 796 & 100,0 & 1.674 & 100,0 & \\
\hline Situação conjugal & & & & & & & 0,000 \\
\hline Solteiro/viúvo/separado & 795 & 65,3 & 544 & 50,0 & 1.339 & 58,1 & \\
\hline Casado/união consensual & 422 & 34,7 & 545 & 50,0 & 967 & 41,9 & \\
\hline Total $^{\mathrm{b}}$ & 1.217 & 100,0 & 1.089 & 100,0 & 2.306 & 100,0 & \\
\hline Possui deficiência ou transtorno ${ }^{c}$ & & & & & & & 0,142 \\
\hline $\operatorname{Sim}$ & 302 & 22,9 & 236 & 20,5 & 538 & 21,8 & \\
\hline Não & 1.014 & 77,1 & 915 & 79,5 & 1.929 & 78,2 & \\
\hline Total $^{\mathrm{b}}$ & 1.316 & 100,0 & 1.151 & 100,0 & 2.467 & 100,0 & \\
\hline
\end{tabular}

Fonte: Ministério da Saúde, Secretaria de Vigilância em Saúde, Sistema de Informação de Agravos de Notificação (SINAN). ${ }^{a}$ Excluídos os registros com raça/cor da pele amarela $(n=18)$ e indígena $(n=21)$. ${ }^{\text {bos }}$ totais divergem devido a dados faltantes (em branco/ignorado). 'Inclui deficiência física, mental, visual, auditiva, transtorno mental, de comportamento e outras deficiências/síndromes. 
entre as vítimas do sexo feminino (22,3\%). O principal desfecho dos episódios de violência foi a alta $(90,4 \%)$, com maior frequência entre as mulheres (92,5\%). Aproximadamente 6\% dos casos evoluíram ao óbito por violência, com proporção maior entre os idosos do sexo masculino $(8,2 \%)$. Todas as características da ocorrência apresentaram diferenças significativas entre homens e mulheres (Tabela 2).

A Tabela 3 mostra a distribuição das notificações de violência contra idosos segundo tipo de violência e meio de agressão por sexo da vítima. Os tipos de violência mais relatados foram: abu- so físico $(67,7 \%)$, violência psicológica $(29,1 \%)$ e negligência $(27,8 \%)$. Em menor proporção, foram referidos casos de violência financeira $(7,9 \%)$, abuso sexual $(3,7 \%)$ e tortura $(3,3 \%)$. Com exceção do abuso físico, significativamente mais frequente entre os homens, os casos de violência psicológica, negligência, financeira, sexual e tortura apresentaram frequência significativamente maior entre as mulheres. Os meios mais utilizados para efetivar as agressões contra idosos foram força corporal $(47,8 \%)$ e ameaça $(16,6 \%)$, com distribuição proporcional significativamente superior entre as mulheres. Também foram relata-

Tabela 2. Notificações de violência contra idosos segundo características da ocorrência por sexo. Brasil, 2010.

\begin{tabular}{|c|c|c|c|c|c|c|c|}
\hline \multirow[b]{2}{*}{ Características } & \multicolumn{2}{|c|}{$\begin{array}{c}\text { Feminino } \\
(\mathrm{N}=1.880 \\
52,3 \%)\end{array}$} & \multicolumn{2}{|c|}{$\begin{array}{c}\text { Masculino } \\
(\mathrm{N}=1.713 \\
47,7 \%)\end{array}$} & \multicolumn{2}{|c|}{$\begin{array}{c}\text { Total } \\
(\mathrm{N}=3.593 \\
100 \%)\end{array}$} & \multirow[b]{2}{*}{ p-valo } \\
\hline & $\mathbf{N}$ & $\%$ & $\mathbf{N}$ & $\%$ & $\mathbf{N}$ & $\%$ & \\
\hline Ocorrência no domicílioa & & & & & & & 0,000 \\
\hline Sim & 1.389 & 86,9 & 911 & 69,0 & 2.300 & 78,8 & \\
\hline Não & 209 & 13,1 & 410 & 31,0 & 619 & 21,2 & \\
\hline Total $^{\mathrm{b}}$ & 1.598 & 100,0 & 1.321 & 100,0 & 2.919 & 100,0 & \\
\hline Violência de repetição & & & & & & & 0,000 \\
\hline $\operatorname{Sim}$ & 766 & 62,5 & 439 & 42,8 & 1.205 & 53,6 & \\
\hline Não & 459 & 37,5 & 586 & 57,2 & 1.045 & 46,4 & \\
\hline Total $^{\mathrm{b}}$ & 1.225 & 100,0 & 1.025 & 100,0 & 2.250 & 100,0 & \\
\hline Lesão autoprovocada & & & & & & & 0,000 \\
\hline Sim & 103 & 8,5 & 158 & 13,1 & 261 & 10,8 & \\
\hline Não & 1.111 & 91,5 & 1050 & 86,9 & 2.161 & 89,2 & \\
\hline Total $^{\mathrm{b}}$ & 1.214 & 100,0 & 1.208 & 100,0 & 2.422 & 100,0 & \\
\hline Natureza da lesão & & & & & & & 0,000 \\
\hline Corte/laceração/amputação & 210 & 14,8 & 525 & 35,8 & 735 & 25,5 & \\
\hline Contusão/entorse/luxação & 373 & 26,3 & 279 & 19,0 & 652 & 22,6 & \\
\hline Fratura/traumas & 126 & 8,9 & 186 & 12,7 & 312 & 10,8 & \\
\hline Outros $^{c}$ & 277 & 19,6 & 226 & 15,4 & 503 & 17,4 & \\
\hline Sem lesão & 431 & 30,4 & 250 & 17,1 & 681 & 23,6 & \\
\hline Total $^{\mathrm{b}}$ & 1.417 & 100,0 & 1.466 & 100,0 & 2.883 & 100,0 & \\
\hline Parte do corpo atingida & & & & & & & 0,000 \\
\hline Cabeça/pescoço & 336 & 36,8 & 588 & 50,2 & 924 & 44,3 & \\
\hline Tórax/abdome/pelve & 139 & 15,2 & 170 & 14,5 & 309 & 14,8 & \\
\hline Membros superiores & 204 & 22,3 & 202 & 17,2 & 406 & 19,5 & \\
\hline Membros inferiores & 87 & 9,5 & 72 & 6,1 & 159 & 7,6 & \\
\hline Múltiplas regiões & 147 & 16,1 & 140 & 11,9 & 287 & 13,8 & \\
\hline Total $^{\mathrm{b}}$ & 913 & 100,0 & 1.172 & 100,0 & 2.085 & 100,0 & \\
\hline Evolução & & & & & & & 0,000 \\
\hline Alta & 1.167 & 92,5 & 1.160 & 88,3 & 2.327 & 90,4 & \\
\hline Evasão & 30 & 2,4 & 19 & 1,4 & 49 & 1,9 & \\
\hline Óbito por violência & 45 & 3,6 & 108 & 8,2 & 153 & 5,9 & \\
\hline Óbito por outras causas & 19 & 1,5 & 26 & 2,0 & 45 & 1,7 & \\
\hline Total $^{\mathrm{b}}$ & 1.261 & 100,0 & 1.313 & 100,0 & 2.574 & 100,0 & \\
\hline
\end{tabular}

Fonte: Ministério da Saúde, Secretaria de Vigilância em Saúde, Sistema de Informação de Agravos de Notificação (SINAN). anclui

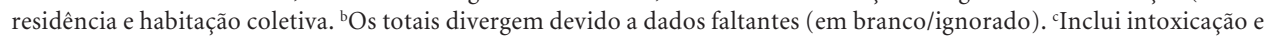
queimadura $(\mathrm{n}=156)$. 
Tabela 3. Notificações de violência contra idosos segundo tipo de violência e meio de agressão por sexo. Brasil, 2010.

\begin{tabular}{|c|c|c|c|c|c|c|c|}
\hline \multirow[b]{2}{*}{ Características } & \multicolumn{2}{|c|}{$\begin{array}{c}\text { Feminino } \\
(\mathrm{N}=1.880 \\
52,3 \%)\end{array}$} & \multicolumn{2}{|c|}{$\begin{array}{c}\text { Masculino } \\
(\mathrm{N}=1.713 ; \\
\quad 47,7 \%)\end{array}$} & \multicolumn{2}{|c|}{$\begin{array}{c}\text { Total } \\
(\mathrm{N}=3.593 \\
100 \%)\end{array}$} & \multirow[b]{2}{*}{ p-valor } \\
\hline & $\mathbf{N}$ & $\%$ & $\mathbf{N}$ & $\%$ & $\mathbf{N}$ & $\%$ & \\
\hline \multicolumn{8}{|l|}{ Tipo de violênciab } \\
\hline Física & 1.046 & 61,3 & 1.199 & 74,6 & 2.245 & 67,7 & 0,000 \\
\hline Psicológica/moral & 636 & 39,1 & 263 & 18,0 & 899 & 29,1 & 0,000 \\
\hline Negligência/abandono & 501 & 30,6 & 366 & 24,7 & 867 & 27,8 & 0,000 \\
\hline Financeira/econômica & 155 & 9,7 & 85 & 5,8 & 240 & 7,9 & 0,000 \\
\hline Sexual & 96 & 6,0 & 17 & 1,2 & 113 & 3,7 & 0,000 \\
\hline Tortura & 66 & 4,2 & 35 & 2,4 & 101 & 3,3 & 0,007 \\
\hline Intervenção legal $^{c}$ & 7 & 0,4 & 3 & 0,2 & 10 & 0,3 & - \\
\hline Outros & 97 & 6,4 & 88 & 6,3 & 185 & 6,4 & 0,979 \\
\hline \multicolumn{8}{|l|}{ Meio de agressão ${ }^{\mathrm{b}}$} \\
\hline Força corporal & 775 & 49,6 & 685 & 45,9 & 1.460 & 47,8 & 0,039 \\
\hline Ameaça & 356 & 23,8 & 128 & 9,1 & 484 & 16,6 & 0,000 \\
\hline Objeto perfurocortante & 87 & 5,8 & 199 & 13,9 & 286 & 9,8 & 0,000 \\
\hline Objeto contundente & 93 & 6,2 & 165 & 11,6 & 258 & 8,8 & 0,000 \\
\hline Arma de fogo & 21 & 1,4 & 110 & 7,7 & 131 & 4,5 & 0,000 \\
\hline Enforcamento & 52 & 3,5 & 60 & 4,2 & 112 & 3,8 & 0,274 \\
\hline Envenenamento & 45 & 3,0 & 46 & 3,2 & 91 & 3,1 & 0,695 \\
\hline Substância/objeto quente & 21 & 1,4 & 14 & 1,0 & 35 & 1,2 & 0,307 \\
\hline Outro & 269 & 19,1 & 217 & 16,5 & 486 & 17,8 & 0,079 \\
\hline
\end{tabular}

Fonte: Ministério da Saúde, Secretaria de Vigilância em Saúde, Sistema de Informação de Agravos de Notificação (SINAN). a totais divergem devido a dados faltantes (em branco/ignorado). 'Não totaliza $100 \%$, pois trata-se de variável com múltipla resposta. 'Não foi calculado o teste do qui-quadrado $\left(\chi^{2}\right)$ e o p-valor devido a existência de célula com valor menor que cinco.

dos como meios de agressão objeto perfurocortante $(9,8 \%)$, objeto contundente $(8,8 \%)$ e arma de fogo $(4,5 \%)$, com frequência significativamente maior entre os homens.

A maioria dos eventos de violência contra idosos foi causada por agressores do sexo masculino $(66,4 \%)$, com proporção significativamente mais elevada entre os homens $(72,1 \%)$. Os agressores eram, em sua maioria, filhos $(32,2 \%)$, pessoas desconhecidas $(15,6 \%)$, parceiros conjugais $(13,9 \%)$ e outras pessoas conhecidas pela vítima $(11,8 \%)$. Houve diferença significativa na distribuição dos agressores segundo o sexo da vítima: as mulheres foram violentadas mais frequentemente por filhos e parceiros conjugais do que os homens. Estes, por sua vez, foram vítimas de pessoas desconhecidas e de seu círculo de convívio em maior proporção do que o observado entre as idosas. A suspeita de que o agressor fez uso de bebida alcoólica foi apontada por 40,4\% das vítimas, sendo significativamente mais relatada pelas mulheres (44\%) do que pelos homens $(36,1 \%)$ (Tabela 4).
Na Tabela 5, apresentam-se a frequência, proporção e razão de proporções dos tipos mais notificados de violência contra idosos segundo características selecionadas. A violência física foi significativamente mais frequente no sexo masculino (RP $=0,82)$, no grupo com 60 a 69 anos de idade $(\mathrm{RP}=$ $1,36)$, ocorrida fora do domicílio ( $R P=0,77)$, praticada por agressores que não eram os filhos $(\mathrm{RP}=$ 0,60), com suspeita de ingestão de bebida alcoólica $(\mathrm{RP}=1,26)$. A violência psicológica foi duas vezes mais frequente entre idosas $(\mathrm{RP}=2,17)$, ocorrida no domicílio $(\mathrm{RP}=2,00)$, infligida pelos filhos ( $\mathrm{RP}$ $=1,59)$, com suspeita de uso de bebida alcoólica $(\mathrm{RP}=1,60)$ e de maneira crônica $(\mathrm{RP}=2,51)$. A negligência predominou no sexo feminino $(\mathrm{RP}=$ $1,24)$, no grupo a partir de 70 anos $(R P=0,34)$, ocorrida no domicílio $(\mathrm{RP}=2,07)$, perpetrada pelos filhos $(\mathrm{RP}=3,39)$, sem suspeita de ingestão de bebida alcoólica $(\mathrm{RP}=0,4)$, de maneira recorrente $(\mathrm{RP}=3,51)$. A violência sexual foi cinco vezes mais comum no sexo feminino ( $\mathrm{RP}=5,21$ ), por agressores que não eram os filhos $(R P=0,25)$, mas que consumiram bebida alcoólica $(\mathrm{RP}=2,11)$. 
Tabela 4. Notificações de violência contra idosos segundo características do agressor por sexo. Brasil, 2010.

\begin{tabular}{|c|c|c|c|c|c|c|c|}
\hline \multirow[b]{2}{*}{ Características } & \multicolumn{2}{|c|}{$\begin{array}{c}\text { Feminino } \\
(\mathrm{N}=1.880 \\
52,3 \%)\end{array}$} & \multicolumn{2}{|c|}{$\begin{array}{c}\text { Masculino } \\
(\mathrm{N}=1.713 \\
\quad 47,7 \%)\end{array}$} & \multicolumn{2}{|c|}{$\begin{array}{c}\text { Total } \\
(\mathrm{N}=3.593 \\
100 \%)\end{array}$} & \multirow[b]{2}{*}{ p-valor } \\
\hline & $\mathbf{N}$ & $\%$ & $\mathbf{N}$ & $\%$ & $\mathbf{N}$ & $\%$ & \\
\hline Sexo do agressor & & & & & & & 0,000 \\
\hline Masculino & 871 & 61,6 & 846 & 72,1 & 1.717 & 66,4 & \\
\hline Feminino & 378 & 26,8 & 193 & 16,5 & 571 & 22,1 & \\
\hline Ambos & 164 & 11,6 & 134 & 11,4 & 298 & 11,5 & \\
\hline Total $^{\mathrm{a}}$ & 1.413 & 100,0 & 1.173 & 100,0 & 2.586 & 100,0 & \\
\hline \multicolumn{8}{|l|}{ Agressor ${ }^{\mathrm{b}}$} \\
\hline Filhos & 533 & 37,5 & 324 & 26,1 & 857 & 32,2 & 0,000 \\
\hline Desconhecidos & 150 & 10,8 & 260 & 21,0 & 410 & 15,6 & 0,000 \\
\hline Parceiros $^{c}$ & 269 & 19,1 & 99 & 8,0 & 368 & 13,9 & 0,000 \\
\hline Conhecidos & 127 & 9,2 & 181 & 14,6 & 308 & 11,8 & 0,000 \\
\hline Irmãos & 50 & 3,7 & 50 & 4,1 & 100 & 3,9 & 0,551 \\
\hline Pais $^{\mathrm{d}}$ & 17 & 1,2 & 12 & 1,0 & 29 & 1,1 & 0,538 \\
\hline Outros & 505 & 34,1 & 442 & 33,0 & 947 & 33,6 & 0,559 \\
\hline Suspeita de consumo de bebida alcoólica & & & & & & & 0,000 \\
\hline Sim & 460 & 44,0 & 317 & 36,1 & 777 & 40,4 & \\
\hline Não & 585 & 56,0 & 560 & 63,9 & 1.145 & 59,6 & \\
\hline Total $^{\mathrm{a}}$ & 1.045 & 100,0 & 877 & 100,0 & 1.922 & 100,0 & \\
\hline
\end{tabular}

Fonte: Ministério da Saúde, Secretaria de Vigilância em Saúde, Sistema de Informação de Agravos de Notificação (SINAN). as

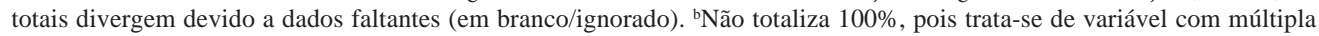
resposta. 'Inclui cônjuge, ex-cônjuge, namorado(a), ex-namorado(a). ${ }^{\mathrm{I} I n c l u i ~ p a i, ~ m a ̃ e, ~ p a d r a s t o, ~ m a d r a s t a . ~}$

\section{Discussão}

O presente estudo apresenta, de maneira inédita, as primeiras análises acerca dos casos de violência contra idosos notificados no âmbito dos serviços de saúde do Brasil em 2010. Revela, ainda, importantes diferenciais do perfil epidemiológico da violência contra o idoso segundo gênero, permitindo conhecer com mais detalhes aspectos relacionados à vítima, ao evento notificado $\mathrm{e}$ ao agressor.

No Brasil, ainda são escassos os estudos que estimam a prevalência de violência contra idosos ou que descrevam os aspectos epidemiológicos desses casos de maneira padronizada para o país. Segundo dados do Suplemento de Saúde da Pesquisa Nacional por Amostra de Domicílios $(\mathrm{PNAD})^{16}$, realizada em 2008, a prevalência de violência entre idosos foi estimada em 1,4\%, independente do tipo. Um inquérito de base populacional realizado com idosos de Niterói (Rio de Janeiro $)^{17}$ identificou uma prevalência de violência física de $10,1 \%$ e $7,9 \%$ dos entrevistados referiram ter sofrido violência grave no ano anterior à pes- quisa. Em revisão sistemática sobre a prevalência de maus-tratos na terceira idade, Espíndola e Blay $^{18}$ encontraram estudos conduzidos em diversas regiões do mundo, principalmente nos Estados Unidos e na Europa, que apontavam a prevalência de abuso físico na população idosa variando de 1,2\% (Holanda) a 18\% (Finlândia).

$\mathrm{Na}$ presente casuística, predominaram as vítimas do sexo feminino, metade dos idosos apresentava 60 a 69 anos de idade, e a maioria era constituída de brancos, com até 8 anos de estudo, sem companhia marital, com local de ocorrência na residência, além da identificação de violência de repetição. Achados semelhantes foram encontrados em diversos outros estudos ${ }^{19-21}$, os quais apontam as mulheres idosas, com baixa escolaridade e solteiras, as vítimas mais vulneráveis à violência.

Entretanto, em estudo transversal realizado no Instituto de Medicina Legal do Recife (Pernambuco $)^{21}$ identificou-se que a maioria dos idosos vítimas de violência era do sexo masculino, pardos, casados e com idade entre 60 e 69 anos. Porém, o estudo também revelou a residência 
Tabela 5. Número de casos (n), proporção (\%) e razão de proporções (RP) de violência contra idosos segundo características selecionadas por tipo de violência. Brasil, 2010.

\begin{tabular}{|c|c|c|c|c|c|c|c|c|}
\hline \multirow[b]{3}{*}{ Características } & \multicolumn{8}{|c|}{ Tipo de violência } \\
\hline & \multicolumn{4}{|c|}{ Física } & \multicolumn{4}{|c|}{ Psicológica } \\
\hline & $\mathrm{n}^{\mathrm{a}}$ & $\%$ & RP & (IC95\%) & $\mathrm{n}^{\mathrm{a}}$ & $\%$ & $\mathrm{RP}$ & (IC95\%) \\
\hline \multicolumn{9}{|l|}{ Sexo } \\
\hline Feminino & 1.046 & 61,3 & 0,82 & $(0,78-0,86)$ & 636 & 39,1 & 2,17 & $(1,92-2,46)$ \\
\hline Masculino & 1.199 & 74,6 & 1 & - & 263 & 18,0 & 1 & - \\
\hline \multicolumn{9}{|l|}{ Faixa etária (anos) } \\
\hline $60-69$ & 1.306 & 78,0 & 1,36 & $(1,30-1,43)$ & 444 & 28,9 & 0,99 & $(0,88-1,10)$ \\
\hline$\geq 70$ & 939 & 57,3 & 1 & - & 455 & 29,3 & 1 & - \\
\hline \multicolumn{9}{|l|}{ Ocorrência no domicílio } \\
\hline Sim & 1.330 & 60,6 & 0,77 & $(0,73-0,82)$ & 769 & 36,3 & 2,00 & $(1,66-2,41)$ \\
\hline Não & 476 & 78,3 & 1 & - & 102 & 18,1 & 1 & - \\
\hline \multicolumn{9}{|l|}{ Agressão pelos filhos } \\
\hline Sim & 362 & 44,7 & 0,60 & $(0,56-0,65)$ & 334 & 42,1 & 1,59 & $(1,42-1,78)$ \\
\hline Não & 1.328 & 74,2 & 1 & - & 470 & 26,5 & 1 & - \\
\hline \multicolumn{9}{|c|}{ Agressor consumiu bebida alcoólica } \\
\hline Sim & 586 & 77,9 & 1,26 & $(1,19-1,34)$ & 321 & 44,1 & 1,60 & $(1,41-1,81)$ \\
\hline Não & 689 & 61,7 & 1 & - & 295 & 27,6 & 1 & - \\
\hline \multicolumn{9}{|l|}{ Violência de repetição } \\
\hline Sim & 684 & 59,0 & 0,71 & $(0,67-0,75)$ & 556 & 48,6 & 2,51 & $(2,18-2,89)$ \\
\hline \multirow[t]{2}{*}{ Não } & 852 & 83,0 & 1 & - & 188 & 19,4 & 1 & - \\
\hline & \multicolumn{4}{|c|}{ Negligência } & \multicolumn{4}{|c|}{ Sexual } \\
\hline Características & $\mathrm{n}^{\mathrm{a}}$ & $\%$ & RP & (IC95\%) & $\mathrm{n}^{\mathrm{a}}$ & $\%$ & RP & (IC95\%) \\
\hline \multicolumn{9}{|l|}{ Sexo } \\
\hline Feminino & 501 & 30,6 & 1,24 & $(1,11-1,39)$ & 96 & 6,0 & 5,21 & $(3,12-8,68)$ \\
\hline Masculino & 366 & 24,7 & 1 & - & 17 & 1,2 & 1 & - \\
\hline \multicolumn{9}{|l|}{ Faixa etária (anos) } \\
\hline $60-69$ & 211 & 13,8 & 0,34 & $(0,29-0,39)$ & 65 & 4,3 & 1,37 & $(0,95-1,98)$ \\
\hline$\geq 70$ & 656 & 41,2 & 1 & - & 48 & 3,1 & 1 & - \\
\hline \multicolumn{9}{|l|}{ Ocorrência no domicílio } \\
\hline Sim & 710 & 33,3 & 2,07 & $(1,70-2,53)$ & 79 & 3,8 & 0,85 & $(0,55-1,32)$ \\
\hline Não & 90 & 16,0 & 1 & - & 25 & 4,5 & 1 & - \\
\hline \multicolumn{9}{|l|}{ Agressão pelos filhos } \\
\hline Sim & 421 & 52,0 & 3,39 & $(2,98-3,85)$ & 9 & 1,2 & 0,25 & $(0,13-0,49)$ \\
\hline Não & 274 & 15,3 & 1 & - & 83 & 4,7 & 1 & - \\
\hline \multicolumn{9}{|c|}{ Agressor consumiu bebida alcoólica } \\
\hline Sim & 94 & 13,2 & 0,40 & $(0,33-0,49)$ & 38 & 5,4 & 2,11 & $(1,30-3,42)$ \\
\hline Não & 352 & 32,9 & 1 & - & 27 & 2,5 & 1 & - \\
\hline \multicolumn{9}{|l|}{ Violência de repetição } \\
\hline Sim & 381 & 33,6 & 3,51 & $(2,85-4,34)$ & 26 & 2,4 & 0,35 & $(0,22-0,54)$ \\
\hline Não & 92 & 9,6 & 1 & - & 66 & 6,8 & 1 & - \\
\hline
\end{tabular}

Fonte: Ministério da Saúde, Secretaria de Vigilância em Saúde, Sistema de Informação de Agravos de Notificação (SINAN). ${ }^{\circ}$ Os totais divergem devido a dados faltantes (em branco/ignorado). IC95\%: intervalo de confiança de 95\%. As diferenças estatisticamente significantes apresentam-se em negrito.

como o principal local de ocorrência do evento violento. Um dos argumentos que explicaria a maior frequência de situações de violência no domicílio, tanto entre homens como mulheres idosas, seria o fenômeno do choque de gerações, permeado por disputas por espaço físico e também por dificuldades financeiras na família ${ }^{22}$.

A literatura ${ }^{21}$ mostra que as lesões em idosos resultantes de situações de violência apresentam gravidade leve, acometendo mais de uma parte 
do corpo, predominantemente braços e ombro essas características não apresentaram diferenças significativas entre homens e mulheres. Todavia, no presente estudo com os dados oriundos do Sinan Net, foram evidentes as diferenças entre idosos e idosas quanto à natureza da lesão: as lesões de corte/laceração/amputação foram mais frequentes no sexo masculino e a região de cabeça e pescoço, a mais atingida.

A força corporal foi o meio de agressão mais utilizado em ambos os sexos, corroborando com achados de outros estudos ${ }^{21}$. Porém, a ameaça foi maior entre as mulheres. Estes dados podem estar relacionados à predominância de lesões leves, resultando em evolução para alta na maioria dos idosos deste estudo, não obstante a ocorrência de alguns óbitos em decorrência da violência, principalmente no sexo masculino. Uma hipótese para este fato é a provável associação com a utilização de objetos/arma de fogo em maior proporção contra as vítimas do sexo masculino, o que pode ser demonstrado pelo crescente aumento proporcional de homicídios entre idosos do sexo masculino nas últimas décadas ${ }^{8}$.

$\mathrm{Na}$ presente análise, as mulheres foram agredidas por filhos e parceiros conjugais, enquanto que os homens sofreram violência perpetrada por desconhecidos. Tal constatação é confirmada pela literatura ${ }^{21,23}$, que aponta a maior participação de agressores do sexo masculino e conhecidos da vítima quando se investiga a epidemiologia da violência contra idosos. No presente trabalho, as mulheres foram mais frequentemente vítimas de violência psicológica, negligência, financeira, sexual e tortura do que os homens. Um estudo realizado na Finlândia demonstrou que as violências psicológica, financeira e a negligência também foram mais prevalentes no sexo feminino. $\mathrm{O}$ abuso físico também foi predominante entre as idosas finlandesas ${ }^{18}$. Entretanto, os nossos dados demonstraram maior proporção de violência física entre os homens.

O relato de suspeita de uso de álcool pelo agressor foi mais frequente entre as idosas, e os seguintes tipos de violência foram relacionados ao consumo de álcool: violência física, negligência e abuso sexual. Problemas com uso de álcool são associados à violência contra os idosos e os agressores provavelmente apresentam distúrbios de personalidade, abuso de outras drogas e dificuldades na relação idoso-cuidador ${ }^{6}$. Limitações cognitivas e físicas, isolamento social, fatores culturais como a aceitação da violência, discriminação de pessoas idosas, aglomeração na residência, ser portador de doença crônica, fragi- lidade nas relações familiares e histórico de violência familiar são reconhecidas como fatores de risco para o idoso com relação a situações de violência ${ }^{8,24}$.

Como limitação do presente estudo, apontase o pequeno número de notificações, dificultando a elaboração de um panorama geral sobre a situação de violência contra os idosos no país. Em 2010, apenas $524(<10 \%)$ dos mais de 5,5 mil municípios brasileiros ${ }^{3}$ apresentaram notificações de casos de violência no Sinan Net. O número de notificações por estado também foi pequeno e inexistente no estado do Pará naquele ano. Essa constatação evidencia a expressiva subnotificação da violência na população de idosos, pois outras fontes de informação como a mídia denunciam a ocorrência desse agravo rotineiramente. Dentre as razões descritas para a subnotificação, citamse a dificuldade de denúncia da violência doméstica contra os idosos, o despreparo dos profissionais de saúde para investigar os casos (falta de capacitação e conhecimento de protocolos de investigação), a infraestrutura deficiente de atendimento e fragilidade das redes de apoio ${ }^{22,25-27}$.

Diversas iniciativas, como o Disque Idoso em Sobral (Ceará) ${ }^{22}$, tem contribuído para aumentar os canais de comunicação entre a população e os serviços de saúde, principalmente em situações que envolvem violência contra idosos. Entretanto, a construção de redes de apoio efetivas para o atendimento integral dos idosos requer o comprometimento do Estado e da sociedade civil $^{26}$. A Organização Mundial de Saúde ${ }^{6}$ reconhece a notificação como uma ferramenta de intervenção contra a violência. Logo, a redução da subnotificação bem como a melhoria da qualidade dos registros é essencial para o conhecimento da magnitude das situações de violência vivenciadas pelos idosos, a implementação de medidas preventivas e de apoio às vítimas e a aplicação de modelos mais resolutivos ${ }^{28,29}$.

A partir dos resultados aqui apresentados, conclui-se que a violência contra a pessoa idosa foi mais prevalente no sexo feminino, pessoas sem convívio marital, ocorrida no domicílio e perpetrada por pessoas do convívio das vítimas. Mesmo com necessidade de aprimoramento, os dados do Sistema de Vigilância de Violências e Acidentes geram informações que contribuem para avançar na capacitação dos profissionais de saúde, consolidação e melhoria das redes de apoio às vítimas, monitoramento e prevenção dos casos de violência contra idosos. Para dimensionar a real magnitude do problema da violência contra os idosos no Brasil, faz-se necessá- 
rio intensificar as notificações e o primeiro passo

é fornecer meios para romper o silêncio.

\section{Colaboradores}

MDM Mascarenhas, SSCA Andrade e ACM Neves trabalharam na concepção, delineamento do estudo, análise dos dados e redação do manuscrito. AAG Pedrosa, MMA Silva e DC Malta contribuíram na interpretação dos resultados, revisão e crítica do manuscrito. Todos os autores aprovaram a versão final do manuscrito.

\section{Referências}

1. United Nations (UN). Department of Economic and Social Affairs. Population Division. World Population Ageing 2009. New York: UN; 2009.

2. Carvalho JAM, Rodríguez-Wong LL. A transição da estrutura etária brasileira na primeira metade do século XXI. Cad Saude Publica 2008; 24(3):597-605.

3. Instituto Brasileiro de Geografia e Estatística (IBGE). Censo [dados na Internet]. Rio de Janeiro: IBGE; 2010 [acessado 2012 jun 30]. Disponível em: http:/ /www.ibge.gov.br

4. World Health Organization (WHO). Envelhecimento ativo: uma politica de saúde. Brasília: Organização Pan-Americana da Saúde; 2005.

5. Perel-Levin S. Discussing screening for elder abuse at Primary Health Care level. Geneva: World Health Organization; 2008.

6. Krug EG, Dahlberg LL, Mercy JA, Zwi AB, Lozano R. World report on violence and health. Geneva: World Health Organization; 2002.

7. International Network for the Prevention of Elder Abuse (INPEA) [site na Internet]. Vancouver: INPEA; 2010 [acessado 2010 set 20]. Disponível em: http://www.inpea.net/home.html

8. Minayo MCS. Violência contra idosos: relevância para um velho problema. Cad Saude Publica 2003; 19(3):783-791

9. Mascarenhas MDM, Monteiro RA, Sá NNB, Gonzaga LAA, Neves ACM, Roza DL, Silva MMA, Duarte EC, Malta DC. Epidemiologia das causas externas no Brasil: mortalidade por acidentes e violências no período de 2000 a 2009. In: Brasil. Ministério da Saúde (MS). Secretaria de Vigilância em Saúde. Saúde Brasil 2010: uma análise da situação de saúde e de evidências selecionadas de impacto de ações de vigilância em saúde. Brasília: MS; 2011.

10. Gawryszewski VP, Silva MMA, Malta DC, Mascarenhas MDM, Costa VC, Matos SG, Moraes Neto OL, Monteiro RA, Carvalho CG, Magalhães ML A proposta da rede de serviços sentinela como estratégia da vigilância de violências e acidentes. Cien Saude Colet 2006; 11(Supl.):1269-1278.

11. Brasil. Lei $n^{\circ}$. 10.741, de $1^{\circ}$ de outubro de 2003. Dispõe sobre o Estatuto do Idoso e dá outras providências. Diário Oficial da União 2003; 3 out.

12. Brasil. Lei $n^{\circ} .12 .461$, de 26 de julho de 2011. Altera a Lei $\mathrm{n}^{\circ} 10.741$, de $1^{\circ}$ de outubro de 2003 , para estabelecer a notificação compulsória dos atos de violência praticados contra o idoso atendido em serviço de saúde. Diário Oficial da União 2011; 27 jul. 
13. Brasil. Portaria $\mathrm{n}^{\circ}$. 2.472, de 31 de agosto de 2010 . Define as terminologias adotadas em legislação nacional, conforme disposto no Regulamento Sanitário Internacional 2005 (RSI 2005), a relação de doenças, agravos e eventos em Saúde Pública de notificação compulsória em todo o território nacional e estabelecer fluxo, critérios, responsabilidades e atribuições aos profissionais e serviços de saúde. Diário Oficial da União 2010; 1 set.

14. Brasil. Portaria 104, de 25 de janeiro de 2011. Define as terminologias adotadas em legislação nacional, conforme o disposto no Regulamento Sanitário Internacional 2005 (RSI 2005), a relação de doenças, agravos e eventos em Saude Publica de notificação compulsória em todo o território nacional e estabelece fluxo, critérios, responsabilidades e atribuições aos profissionais e serviços de saúde. Diário Oficial da União 2011; 26 jan.

15. Brasil. Ministério da Saúde (MS). Viva: instrutivo de notificação de violência doméstica, sexual e outras violências. Brasília: MS; 2011.

16. Instituto Brasileiro de Geografia e Estatística (IBGE). Pesquisa Nacional por Amostra de Domicílios. Um panorama da Saúde no Brasil: Acesso e Utilização de Serviços, Condições de Saúde e Fatores de Risco e Proteção à Saúde 2008. Rio de Janeiro: IBGE; 2010

17. Moraes CL, Apratto Júnior PC, Reichenheim ME. Rompendo o silêncio e suas barreiras: um inquérito domiciliar sobre a violência doméstica contra idosos em área de abrangência do Programa Médico de Família de Niterói, Rio de Janeiro, Brasil. Cad Saude Publica 2008; 24(10):2289-2300.

18. Espindola CR, Blay SL. Prevalência de maus-tratos na terceira idade: revisão sistemática. Rev Saude Publica 2007; 41(2):301-306.

19. Sanches APRA. Violência doméstica contra idosos no município de São Paulo: Estudo SABE, 2000 [dissertação]. São Paulo: Universidade de São Paulo; 2006.

20. Melo VL, Cunha JOC, Falbo Neto GH. Maus-tratos contra idosos no município de Camaragibe, Pernambuco. Rev Bras Saude Mater Infant 2006; 6(Supl. 1):s43-s48.

21. Abath MB, Leal MCC, Melo Filho DA, Marques APO. Physical abuse of older people reported at the Institute of Forensic Medicine in Recife, Pernambuco State, Brazil. Cad Saude Publica 2010; 26(9):1797-1806.
22. Freitas CASL, Teófilo TJS. Avaliação construtivista, sob uma abordagem integradora e intersetorial, das ações do Projeto Disque Idoso em Sobral (CE, Brasil). Cien Saude Colet 2010; 15(6):2825-2833.

23. Gaioli CCLO, Rodrigues RAP. Occurrence of domestic elder abuse. Rev Latino Am Enfermagem 2006; 16(3):465-470

24. Queiroz ZPV, Lemos NFD, Ramos LR. Fatores potencialmente associados à negligência doméstica entre idosos atendidos em programa de assistência domiciliar. Cien Saude Colet 2010; 15(6):28152824.

25. Souza ER, Minayo MCS. Inserção do tema violência contra a pessoa idosa nas políticas públicas de atenção à saúde no Brasil. Cien Saude Colet 2010; 15(6):2659-2668.

26. Cavalcanti MLT, Souza ER. Percepções de gestores e profissionais de saúde sobre a atenção aos idosos vítimas de violências no município do Rio de Janeiro (RJ, Brasil). Cien Saude Colet 2010; 15(6): 2699-2708.

27. Mello ALSF, Moysés SJ. Análise diagnóstica do atendimento pré-hospitalar para acidentes e violências contra idosos em Curitiba (PR, Brasil). Cien Saude Colet 2010; 15(6):2709-2718.

28. Santos ER, Souza ER, Ribeiro AP, Souza AMM, Lima RTS. Cenário do atendimento aos agravos provocados por acidentes e violência contra idosos na rede SUS de Manaus (AM, Brasil). Cien Saude Colet 2010; 15(6): 2741-2752.

29. Veras R. Vida plena sem violência na maturidade: a busca contemporânea. Cien Saude Colet 2010; 15(6):2671-2673.

Artigo apresentado em 10/06/2012

Aprovado em 04/07/2012

Versão final apresentada em 14/07/2012 